PROCEEDINGS OF THE AMERICAN MATHEMATICAL SOCIETY

Volume 124, Number 11, November 1996

\title{
ON THE EXISTENCE OF MINIMAL SURFACES WITH SINGULAR BOUNDARIES
}

\author{
HOWARD ISERI
}

(Communicated by Peter Li)

\begin{abstract}
In 1931, Jesse Douglas showed that in $\mathbb{R}^{n}$, every set of $k$ rectifiable Jordan curves, with $k \geq 2$, bounds an area-minimizing minimal surface with prescribed topological type if a "condition of cohesion" is satisfied. In this paper, it is established that under similar conditions, this result can be extended to non-Jordan curves.
\end{abstract}

\section{INTRODUCTION}

The standard example illustrating Douglas' theorem is the existence of a minimal surface bounded by two circles. If the circles are sufficiently close together, a catenoid attaining the infimum of area will span the circles, Figure 1. If the circles are sufficiently far apart, only a degenerate surface consisting of two disks connected by an arc can attain the infimum of area, Figure 2. By imagining a narrow tube around the arc in Figure 2, it is clear that there are non-degenerate surfaces whose areas approach the area of the two disks. It is also clear that the area of any surface with a sufficiently small "waist" can be reduced by collapsing the surface into an arc and then into the two disks.

Douglas' condition of cohesion, in some sense, requires that there are surfaces whose areas are arbitrarily close to the infimum but which have waists that do not get arbitrarily small, and this is sufficient to ensure that there is a non-degenerate solution. In the higher genus case, this condition of cohesion will prevent a surface from "degenerating" into a lower genus surface. A proof of Douglas' theorem can be found in $[\mathrm{C}],[\mathrm{T}]$, and $[\mathrm{L}]$.

If the boundary curves are not Jordan, another kind of degenerate solution is possible. This can be illustrated by taking a circle and a figure-eight curve. It is possible that the infimum of area can only be attained by degenerate surfaces consisting of an annulus spanning one loop of the figure-eight and the circle, and a disk spanning the remaining loop of the figure-eight, Figure 3. If this is the case, the area of a non-degenerate surface can be reduced by collapsing the surface into the singular boundary point, Figure 4 . In the non-zero genus case, it is also possible that a curve passing through a "hole" can collapse into a singular boundary point.

Received by the editors May 9, 1995.

1991 Mathematics Subject Classification. Primary 53A10, 49Q05.

Key words and phrases. Plateau's problem, minimal surfaces, singular boundary curves, Douglas condition, condition of cohesion, condition of adhesion.

This work was begun as a graduate student at the University of California, Davis, under the continuing guidance of Professor Joel Hass. 


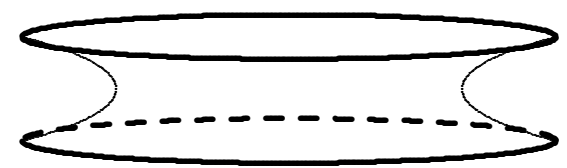

Figure 1. Catenoidal solution

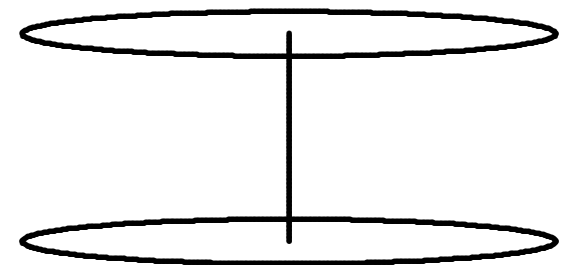

FiguRE 2. Degenerate solution-surface collapsed to an arc

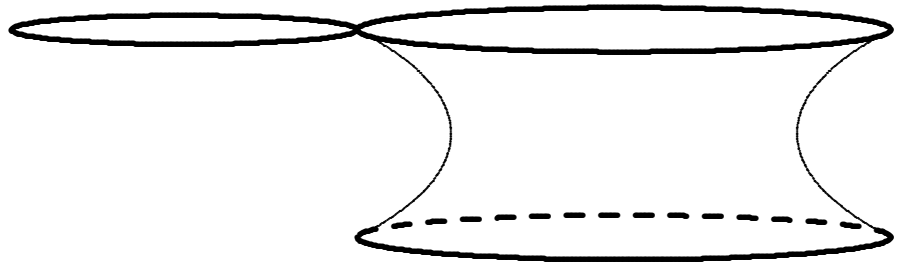

Figure 3. Degenerate solution - disk is pinched off of surface

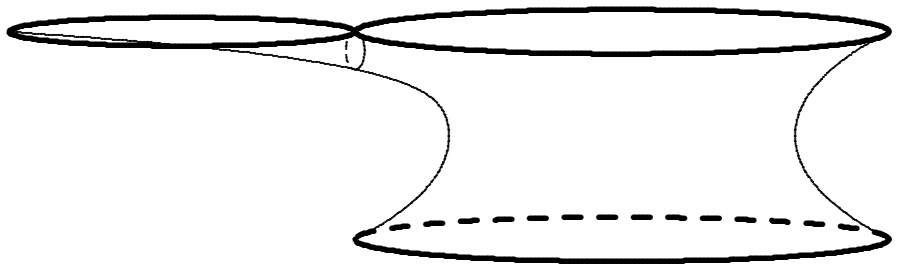

Figure 4. Image of $\psi$

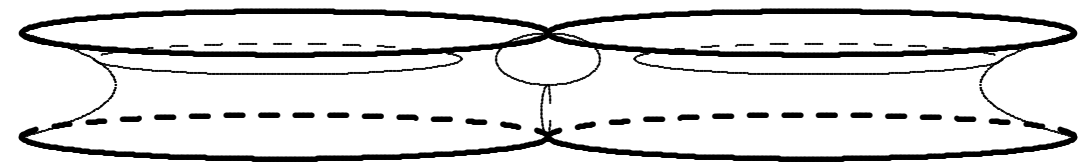

Figure 5. Degenerate genus one surface

Figure 5 shows a degenerate genus one surface with two boundary components whose "interior" is a genus zero surface with three boundary components.

A "condition of adhesion" will be introduced requiring that there are surfaces whose areas approach the infimum so that curves in the surface like the one pictured in Figure 4 do not get arbitrarily small. In Section 3, it will be shown that the Jordan condition in Douglas' theorem can be replaced with this condition of adhesion 
in the zero genus case, and in Section 4, the higher genus case is discussed. It will also be clear that if a solution surface exists, then both the condition of cohesion and the condition of adhesion will be satisfied, and so both conditions are, in fact, necessary.

Furthermore, there is a slightly more restrictive "Douglas condition" which is expressed in terms of area. If $a$ is the infimum of area over all non-degenerate surfaces, and $a^{*}$ is the infimum over all degenerate surfaces, and $a<a^{*}$, then the condition of cohesion is satisfied. In Section 5, it is shown using area arguments in conjunction with the condition of adhesion that certain minimal surfaces must exist.

\section{Preliminary Definitions}

A curve $\gamma$ in $\mathbb{R}^{n}$ will be taken to be a continuous immersion $\gamma: S^{1} \rightarrow \mathbb{R}^{n}$ where $S^{1}$ is the unit circle, and by an immersion it is meant that $\gamma^{-1}(x)$ is totally disconnected for each $x$ in the image of $\gamma$. This is, of course, weaker than the Jordan condition which would correspond to $\gamma$ being a homeomorphism. The length of $\gamma$ will be denoted by $l(\gamma)$, and the curve $\gamma$ is rectifiable if $l(\gamma)<\infty$.

A surface $f$ in $\mathbb{R}^{n}$ (genus zero) will be defined as follows. Take any set of $k-1$ disjoint closed disks contained in the interior of the unit disk. Let $R$ be the closed unit disk with the interiors of the $k-1$ enclosed disks removed. A surface $f$ in $\mathbb{R}^{n}$ will be a map $f: R \rightarrow \mathbb{R}^{n}$ continuous in $R$ and piecewise smooth in the interior.

If $f: R \rightarrow \mathbb{R}^{n}$ is a surface, the boundary of $R$ will consist of $k$ circles denoted $\partial^{1} R, \partial^{2} R, \ldots, \partial^{k} R$. The surface $f$ will be said to be bounded by the $k$ curves $\gamma^{1}, \gamma^{2}, \ldots, \gamma^{k}$ if $\left.f\right|_{\partial^{i} R}$ is a monotonic reparametrization of $\gamma^{i}$ for each $i$. Here, $h: \partial^{i} R \rightarrow S^{1}$ is monotonic if $h^{-1}(x)$ is connected for each $x$ in $S^{1}$.

The Dirichlet integral of a surface $f: R \rightarrow \mathbb{R}^{n}$ is defined to be

$$
D[f]=\int_{R}\left\|f_{u}\right\|^{2}+\left\|f_{v}\right\|^{2} d u d v .
$$

Let $d$ represent the infimum of $D[f]$ over all surfaces bounded by $\gamma^{1}, \ldots, \gamma^{k}$. In general, the area of $f$ is less than or equal to $\frac{1}{2} D[f]$, and it can be shown that if $a$ is the infimum of area, then $a=\frac{1}{2} d$, see $[\mathrm{C}],[\mathrm{L}]$.

\section{The genus Zero CASE}

The purpose of this paper is to establish the existence of an area-minimizing minimal surface given $k$ curves under certain conditions. First of all, there are collections of curves that bound only surfaces of infinite area. These, of course, should be eliminated from consideration, and it is clearly sufficient to do so by considering only rectifiable curves.

There are also two situations where an area-minimizing minimal surface does not exist, and there is only a degenerate solution. The first type of degenerate solution can be eliminated by the condition of cohesion, and the second by the condition of adhesion.

Given a surface $f: R \rightarrow \mathbb{R}^{n}$, consider the set of all Jordan curves $\phi$ in $R$ that do not bound a disk in $R$. Define the cohesion of $f$ to be

$$
\operatorname{co}(f)=\inf _{\phi}\{l(f(\phi))\}
$$


If there is a sequence of surfaces $\left\{f_{i}\right\}$ with $D\left[f_{i}\right] \rightarrow d$, and a $\sigma>0$, such that $\operatorname{co}\left(f_{i}\right)>\sigma$ for all $i$, then it will be said that the condition of cohesion is satisfied.

Consider all simple (but not closed) curves $\psi$ in $R$ such that $\psi^{\circ} \subset R$ and $\partial \psi \subset$ $\partial^{i} R$ for some $i$. The pair of points $\partial \psi$ divide $\partial^{i} R$ into two components, $\psi^{\prime}$ and $\psi^{\prime \prime}$, the labels chosen so that if one of the components bounds a disk in $R$ with $\psi$, then that component is $\psi^{\prime}$, and otherwise, $\psi^{\prime}$ is chosen so that $l\left(f\left(\psi^{\prime}\right)\right) \leq l\left(f\left(\psi^{\prime \prime}\right)\right)$. Define the $\epsilon$-adhesion of $f$ to be

$$
\operatorname{ad}_{\epsilon}(f)=\inf _{\psi}\{l(f(\psi))\}
$$

where the infimum is taken over all $\psi$ with $l\left(f\left(\psi^{\prime}\right)\right)>\epsilon$. If there is a sequence of surfaces $\left\{f_{i}\right\}$ with $D\left[f_{i}\right] \rightarrow d$ satisfying the condition of cohesion, and for each $\epsilon>0$ there is a $\sigma_{\epsilon}>0$ such that $\operatorname{ad}_{\epsilon}\left(f_{i}\right)>\sigma_{\epsilon}$ for all $i$, then it will be said that the condition of adhesion is satisfied.

Theorem 1. Let $\gamma^{1}, \gamma^{2}, \ldots, \gamma^{k}$ be disjoint rectifiable curves in $\mathbb{R}^{n}$, with $k \geq 2$. If the condition of cohesion and the condition of adhesion are satisfied, then there is a minimal surface that bounds $\gamma^{1}, \ldots, \gamma^{k}$, is a branched immersion, and has smallest possible area.

The proof of this theorem will be only a slight modification of the proof for the Jordan case contained in $[\mathrm{C}]$, so the focus here will be on the parts that are different.

Let $\left\{f_{i}\right\}, f_{i}: R_{i} \rightarrow \mathbb{R}^{n}$, be a sequence of surfaces bounded by $\gamma^{1}, \ldots, \gamma^{k}$ with $D\left[f_{i}\right] \rightarrow d$ and satisfying the condition of cohesion as well as the condition of adhesion. The regions $R_{i}$, in general, will not be conformally equivalent, and since $D\left[f_{i}\right]$ depends on the conformal class of $R_{i}$, care must be used in dealing with these regions. The condition of cohesion guarantees the existence of a limiting conformal class, and so it can be assumed that there is a region $R$ consisting of the closed unit disk with $k-1$ disjoint open disks removed, and $R_{i} \rightarrow R,[\mathrm{C}]$.

Let $\left.f_{i}\right|_{\partial R_{i}}$ be denoted by $\partial f_{i}$. In $[\mathrm{C}]$, the requirement that the boundary curves be Jordan is used only to show that the sequence $\left\{\partial f_{i}\right\}$ is equicontinuous. Here, the condition of adhesion will be used to establish this fact. The proof will depend on the following result due to Courant and Lebesgue, [C].

The Courant-Lebesgue Lemma. Let $K$ be a constant and let $f: R \rightarrow \mathbb{R}^{n}$ be a piecewise smooth map with $D[f]<K$. Let $x$ be a point in $R$ and let $C_{\rho}$ be the set of points in $R$ a distance $\rho$ from $x$. If $0<\delta<1$ then there is a $\rho_{0}$ such that $\delta<\rho_{0}<\sqrt{\delta}$ and

$$
l\left(f\left(C_{\rho_{0}}\right)\right)<\frac{1}{4} \sqrt{\frac{K}{\ln (1 / \delta)}} .
$$

Lemma. The sequence $\left\{\partial f_{i}\right\}$ is equicontinuous.

Proof. Let $\partial^{j} f_{i}$ represent the map $\left.f_{i}\right|_{\partial^{j} R_{i}}$, and since $\partial R_{i}$ has only a finite number of components, it is sufficient to show that $\left\{\partial^{j} f_{i}\right\}$ is equicontinuous for each $j$.

Let $\epsilon>0$. It must be shown that there is a $\delta>0$ such that if $x, y \in \partial^{j} R_{i}$ with $|x-y|<\delta$, then $\left|f_{i}(x)-f_{i}(y)\right|<\epsilon$ for each $i$.

Due to the condition of adhesion, it can be assumed that there is a fixed positive number $\sigma_{\epsilon}$ such that $\operatorname{ad}_{\epsilon}\left(f_{i}\right)>\sigma_{\epsilon}$ for all $i$. Furthermore, since $D\left[f_{i}\right] \rightarrow d$, there 
must be a number $K$ such that $D\left[f_{i}\right]<K$ for all $i$. Finally, for $\delta$ sufficiently small,

$$
\frac{1}{4} \sqrt{\frac{K}{\ln (1 / \delta)}}<\sigma_{\epsilon},
$$

and for $\delta<\rho<\sqrt{\delta}, C_{\rho}^{\prime}$ (as defined in the definition of adhesion) and $C_{\rho}$ will bound a disk in $R$.

Let $x, y \in \partial^{j} R_{i}$ be such that $|x-y|<\delta$. By the Courant-Lebesgue Lemma, there is a number $\rho_{i}$ with $\delta<\rho_{i}<\sqrt{\delta}$ such that $C_{\rho_{i}}$ is centered at $x$ and

$$
l\left(f_{i}\left(C_{\rho_{i}}\right)\right)<\frac{1}{4} \sqrt{\frac{K}{\ln (1 / \delta)}}<\sigma_{\epsilon}
$$

for each $i$. Therefore, because of the condition of adhesion,

$$
l\left(f_{i}\left(C_{\rho_{i}}^{\prime}\right)\right)<\epsilon
$$

and since $x, y \in C_{\rho_{i}}^{\prime}$, we have $\left|f_{i}(x)-f_{i}(y)\right|<\epsilon$, and $\left\{\partial f_{i}\right\}$ is equicontinuous.

Since the sequence $\left\{f_{i}\right\}$ is equicontinuous, by Arzela's theorem, it can be assumed, after passing to a subsequence, that $\left\{\partial f_{i}\right\}$ converges uniformly.

Dirichlet's Principle guarantees the existence of a sequence of harmonic surfaces $\left\{f_{i}^{*}\right\}$ satisfying the boundary conditions $\left\{\partial f_{i}\right\}$, and since it is precisely these harmonic surfaces that minimize the Dirichlet integral given these boundary conditions, $D\left[f_{i}^{*}\right] \leq D\left[f_{i}\right]$. Therefore, $\left\{f_{i}^{*}\right\}$ must converge uniformly to a harmonic surface $f$ bounded by $\gamma^{1}, \ldots, \gamma^{k}$ with $D[f]=d$. It can be shown that the area of $f$ is the infimum of area $a=\frac{1}{2} d$, that $f$ is conformal, and is hence a minimal surface, $[\mathrm{C}]$, $[\mathrm{L}]$.

\section{THE NON-ZERO GENUS CASE}

The non-zero genus case is complicated by the fact that the non-zero genus surfaces can no longer be represented as the image of a region in the plane. In [C], the regions $R$ are replaced by Riemann surfaces, and in [T], $R$ is replaced by a manifold with $k$ boundary components. What follows is a discussion of how the arguments in $[\mathrm{T}]$ can be modified to the present situation.

The conformal class of the manifold is an essential consideration, as it is in the plane, with Riemannian metrics on the manifold determining the conformal class, and it is shown in $[\mathrm{T}]$ that the condition of cohesion guarantees a limiting conformal class. In order to apply results for compact manifolds without boundary, a "symmetric surface" $2 M$ is constructed by taking two copies of the manifold $M$ and identifying the boundaries. The properties of a Jordan curve are used twice, first, in showing that the condition of cohesion on $M$ transfers to $2 M$, and second, in showing that the boundary maps are equicontinuous. Working in local coordinates, the arguments establishing equicontinuity are essentially the same as in the zero genus case, so all that is required is to show that the condition of cohesion holds in $2 M$.

Mimicking Lemma 4.1 in [T], let $f: M \rightarrow \mathbb{R}^{n}$ be a surface bounding $\gamma^{1}, \ldots, \gamma^{k}$ with the boundary components $C^{1}, C^{2}, \ldots, C^{k}$ of $M$ being mapped monotonically by $f$. Let $2 M=M \cup S(M)$, where $S$ is the natural "symmetric" map from $M$ to its copy, and extend $f: 2 M \rightarrow \mathbb{R}^{n}$. Suppose that there is a $\sigma>0$, and for each 
$\epsilon>0$ there is a $\sigma_{\epsilon}>0$, such that $\operatorname{co}(f)>\sigma$ and $\operatorname{ad}_{\epsilon}(f)>\sigma_{\epsilon}$. Fix $\epsilon=\frac{1}{2} \sigma$, and let $\rho$ be the minimum distance between the curves $\gamma^{1}, \ldots, \gamma^{k}$.

Lemma. If $\phi$ is a Jordan curve in $2 M$ that does not bound a disk in $2 M$, then

$$
l(f(\phi)) \geq \min \left\{\rho, \sigma_{\epsilon}, \frac{1}{2} \sigma\right\} .
$$

Since $\rho$ is constant, and $\sigma$ and $\sigma_{\epsilon}$ are bounded below by the condition of cohesion and the condition of adhesion, this lemma will show that the condition of cohesion will transfer to $2 M$.

Proof. Suppose $l(f(\phi))<\min \left\{\rho, \sigma_{\epsilon}, \frac{1}{2} \sigma\right\}$. If $\phi$ lies completely in $M$ or $S(M)$, then clearly, $l(f(\phi)) \geq \operatorname{co}(f)>\sigma$, and this is a contradiction. Otherwise, let $\phi_{1}, \phi_{2}, \ldots$ be the maximal subarcs of $\phi$ lying in $S(M)$, and since $l(f(\phi))<\rho$, the $\partial \phi_{i}$ all lie in the same boundary component of $M$. Let $\phi_{1}^{\prime}, \phi_{2}^{\prime}, \ldots$ be the boundary arcs as defined in the definition of adhesion. If each of $\phi_{i}$ and $\phi_{i}^{\prime}$ bound a disk in $S(M)$, then $S\left(\phi_{i}\right)$ and $\phi_{i}^{\prime}$ bound a disk in $M$, and replacing each $\phi_{i}$ with $S\left(\phi_{i}\right)$ yields a curve in $M$ that does not bound a disk, and again $l(f(\phi))>\sigma$. If some $\phi_{i}$ and $\phi_{i}^{\prime}$ do not bound a disk in $S(M)$, then $l\left(f\left(\phi_{i}\right)\right)+l\left(f\left(\phi_{i}^{\prime}\right)\right) \geq \operatorname{co}(f)>\sigma$. Since $l\left(f\left(\phi_{i}\right)\right)<\sigma_{\epsilon}<\operatorname{ad}_{\epsilon}(f)$, it follows that $l\left(f\left(\phi_{i}^{\prime}\right)\right)<\epsilon=\frac{1}{2} \sigma$. Therefore, $l(f(\phi))>l\left(f\left(\phi_{i}\right)>\frac{1}{2} \sigma\right.$, and again, this is a contradiction.

This proves the following.

Theorem 2. Let $\gamma^{1}, \gamma^{2}, \ldots, \gamma^{k}$ be disjoint rectifiable curves in $\mathbb{R}^{n}$, with $k \geq 2$. If a topological type is specified, and the condition of cohesion and the condition of adhesion are satisfied, then there is a minimal surface of the given topological type that bounds $\gamma^{1}, \ldots, \gamma^{k}$, is a branched immersion, and has smallest possible area.

\section{The CONDITION OF ADHESION AND AREA}

While the condition of cohesion and the condition of adhesion are sufficient to guarantee the existence of a minimal surface, given a collection of curves, it is not trivial to verify that these conditions are satisfied. In this section, a connection between area and the condition of adhesion is established, and it is shown that an area condition is sometimes easy to verify.

The condition of adhesion is needed only in the case that at least one of the boundary curves is not Jordan. If a curve $\gamma: S^{1} \rightarrow \mathbb{R}^{n}$ is not Jordan, then there are two points $x, y \in S^{1}$ such that $\gamma(x)=\gamma(y)$. By removing $x$ and $y$ from $S^{1}$, and identifying the boundary points of each of the components, $\gamma$ can be broken into two new curves $\gamma_{a}$ and $\gamma_{b}$.

If $\gamma^{1}, \gamma^{2}, \ldots, \gamma^{k}$ are $k$ curves and $\gamma^{j}$ can be broken into two curves $\gamma_{a}^{j}$ and $\gamma_{b}^{j}$, then any surface bounded by $\gamma^{1}, \gamma^{2}, \ldots, \gamma_{a}^{j}$ and any surface bounded by $\gamma_{b}^{j}, \ldots, \gamma^{k}$ can be joined together to form a surface bounded by $\gamma^{1}, \ldots, \gamma^{k}$ that is degenerate.

This can be illustrated in the annular case in Figure 6, where the interior arc is mapped to the singular boundary point in Figure 3.

Restricting attention to the zero genus case, let $\mathcal{A}\left(\gamma^{1}, \ldots, \gamma^{k}\right)$ be the infimum of area for non-degenerate surfaces bounded by $\gamma^{1}, \ldots, \gamma^{k}, k \geq 1$.

The proof here is an application of the techniques used in $[\mathrm{H}]$ on the existence of least area disks to the situation here with multiple boundary curves. 




FIGURE 6. Interior arc is mapped to the singular boundary point

Theorem 3. If in Theorem 1 the condition of adhesion is not satisfied, then for some $j, \gamma^{j}$ can be broken into $\gamma_{a}^{j}$ and $\gamma_{b}^{j}$ with both non-trivial, and

$$
\mathcal{A}\left(\gamma^{1}, \ldots, \gamma^{k}\right) \leq \mathcal{A}\left(\gamma^{1}, \ldots, \gamma_{a}^{j}\right)+\mathcal{A}\left(\gamma_{b}^{j}, \ldots, \gamma^{k}\right),
$$

(relabeling the $\gamma^{i}$ if necessary).

Proof. As in Theorem 1, there is a sequence $\left\{f_{i}\right\}$, with $D\left[f_{i}\right] \rightarrow d$. Since the condition of adhesion is not satisfied, it can be assumed that there is an $\epsilon>0$ such that $\operatorname{ad}_{\epsilon}\left(f_{i}\right) \rightarrow 0$. Therefore, there is a sequence of $\operatorname{arcs} \psi_{i} \subset R_{i}$ such that $l\left(f_{i}\left(\psi_{i}\right)\right) \rightarrow 0$ and $l\left(f_{i}\left(\psi_{i}^{\prime}\right)\right)>\epsilon$. Since there are only a finite number of boundary curves, it can be assumed that all of the $\psi_{i}^{\prime}$ 's lie in one of the boundary components $\partial^{j} R_{i}$ (noting that $\partial^{j} R_{i} \rightarrow \partial^{j} R$ ) .

Since $\partial^{j} f_{i}$ is a monotonic reparametrization of $\gamma^{j}$, there is a monotonic map $h_{i}: \partial^{j} R_{i} \rightarrow S^{1}$ such that $\gamma^{j} \circ h_{i}=\partial^{j} f_{i}$. Clearly, $h_{i}\left(\psi_{i}^{\prime}\right)$ is an arc in $S^{1}$, and the boundary points of $\psi_{i}^{\prime}$ can be labeled $\partial^{-} \psi_{i}^{\prime}$ and $\partial^{+} \psi_{i}^{\prime}$ so that moving from $h_{i}\left(\partial^{-} \psi_{i}^{\prime}\right)$ towards $h_{i}\left(\partial^{+} \psi_{i}^{\prime}\right)$ along $h_{i}\left(\psi_{i}^{\prime}\right)$ is counterclockwise in $S^{1}$. Since $S^{1}$ is compact, after passing to a subsequence, it can be assumed that $h_{i}\left(\partial^{-} \psi_{i}^{\prime}\right) \rightarrow t^{-}$ and $h_{i}\left(\partial^{+} \psi_{i}^{\prime}\right) \rightarrow t^{+}$for some $t^{-}$and $t^{+}$in $S^{1}$. Since $l\left(f_{i}\left(\psi_{i}\right)\right) \rightarrow 0$, it follows that $\gamma^{j}\left(t^{-}\right)=\gamma^{j}\left(t^{+}\right)$. Break $\gamma^{j}$ into $\gamma_{a}^{j}$ and $\gamma_{b}^{j}$. Furthermore, $l\left(f_{i}\left(\psi_{i}^{\prime}\right)\right)>\epsilon$, and since $\psi_{i}$ and $\psi_{i}^{\prime \prime}$ do not bound a disk in $R_{i}, l\left(f_{i}\left(\psi_{i}^{\prime \prime}\right)\right)>\frac{1}{2} \sigma$ eventually by the condition of cohesion, so $t^{-} \neq t^{+}$and both $\gamma_{a}^{j}$ and $\gamma_{b}^{j}$ are non-trivial.

Since there are only a finite number of boundary components and each $\psi_{i}$ is simple, there are only a finite number of homotopy types (in $R$ ), and it can be assumed that all are homotopic (in $R$ ).

This homotopy class divides $R$ into two components, one corresponding to $\gamma_{a}^{j}$ and the other to $\gamma_{b}^{j}$. Renumber the $\gamma^{i}$ so that $\gamma^{1}, \gamma^{2}, \ldots, \gamma^{j-1}$ lie in the component corresponding to $\gamma_{a}^{j}$, and $\gamma^{j+1}, \ldots, \gamma^{k}$ do not.

Finally, since each arc $\psi_{i}$ divides $f_{i}$ into a surface approximating a surface bounded by $\gamma^{1}, \ldots, \gamma_{a}^{j}$ and a surface approximating a surface bounded by $\gamma_{b}^{j}$, $\ldots, \gamma^{k}$, it follows that

$$
\mathcal{A}\left(\gamma^{1}, \ldots, \gamma_{a}^{j}\right)+\mathcal{A}\left(\gamma_{b}^{j}, \ldots, \gamma^{k}\right) \leq \mathcal{A}\left(\gamma^{1}, \ldots, \gamma^{k}\right) .
$$

Theorem 3 establishes the following area condition on the existence of a solution surface. If the condition of cohesion is satisfied and

$$
\mathcal{A}\left(\gamma^{1}, \ldots, \gamma^{k}\right)<\mathcal{A}\left(\gamma^{1}, \ldots, \gamma_{a}^{j}\right)+\mathcal{A}\left(\gamma_{b}^{j}, \ldots, \gamma^{k}\right)
$$




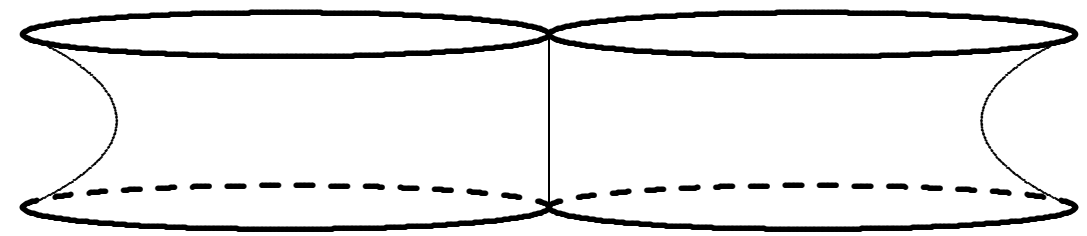

FiguRE 7. Minimal solution surface

for any non-trivial $\gamma_{a}^{j}$ and $\gamma_{b}^{j}$, then the condition of adhesion must be satisfied, and an area-minimizing minimal surface must exist, by Theorem 1 .

To illustrate how this can be used, consider the pair of figure eight curves formed from four circles of radius 1 centered at $(1,0,0),(-1,0,0),(1,0,1 / 10)$, and $(-1,0,1 / 10)$ and lying in the planes $z=0$ and $z=1 / 10$. Since $\mathcal{A}\left(\gamma_{a}^{j}\right), \mathcal{A}\left(\gamma_{b}^{j}\right) \geq \pi$ for any non-trivial $\gamma_{a}^{j}$ and $\gamma_{b}^{j}$, and there is a cylindrical surface with area $4 \pi / 5$, the condition of adhesion is satisfied. Since $\mathcal{A}\left(\gamma^{1}\right)+\mathcal{A}\left(\gamma^{2}\right)=4 \pi$, the Douglas condition, and hence the condition of cohesion, is satisfied. Therefore, there must be an area-minimizing genus zero minimal surface bounded by these two curves (Figure $7)$.

\section{REFERENCES}

[C] R. Courant, Dirichlet's Principle, Interscience, 1950. MR 12:90a

[H] J. Hass, Singular Curves and the Plateau Problem, Int. J. Math. 2 (1) (1991), 1-16. MR 91k:53017

[L] H. B. Lawson, Jr., Lectures on Minimal Submanifolds, Publish or Perish, 1980. MR 82d:53035b

[T] F. Tomi and A. J. Tromba, Existence theorems for minimal surfaces of non-zero genus spanning a contour, Mem. AMS 71 (382) (1988). MR 90a:58032

Department of Mathematics and Computer Information Science, Mansfield UniverSity, Mansfield, Pennsylvania 16933

E-mail address: hiseri@.mnsfld.edu 